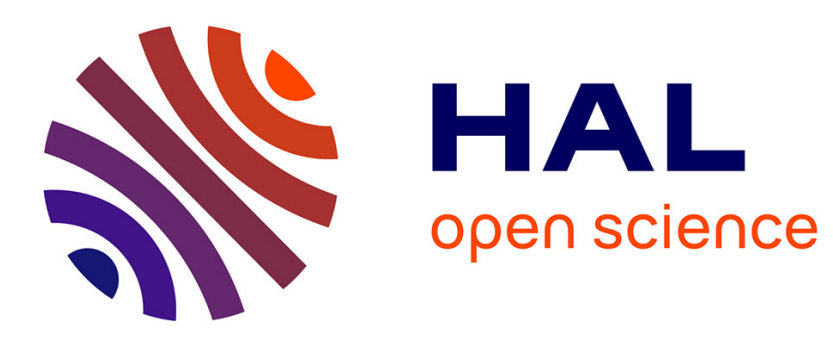

\title{
The Minimum Degree Heuristic and the Minimal Triangulation Process
}

\author{
Anne Berry, Pinar Heggernes, Geneviève Simonet
}

\section{To cite this version:}

Anne Berry, Pinar Heggernes, Geneviève Simonet. The Minimum Degree Heuristic and the Minimal Triangulation Process. WG: Workshop on Graph-Theoretic Concepts in Computer Science, May 2003, Elspeet, Netherlands. pp.58-70, 10.1007/978-3-540-39890-5_6 . lirmm-00191916

\section{HAL Id: lirmm-00191916 https://hal-lirmm.ccsd.cnrs.fr/lirmm-00191916}

Submitted on 26 Nov 2007

HAL is a multi-disciplinary open access archive for the deposit and dissemination of scientific research documents, whether they are published or not. The documents may come from teaching and research institutions in France or abroad, or from public or private research centers.
L'archive ouverte pluridisciplinaire $\mathbf{H A L}$, est destinée au dépôt et à la diffusion de documents scientifiques de niveau recherche, publiés ou non, émanant des établissements d'enseignement et de recherche français ou étrangers, des laboratoires publics ou privés. 


\title{
The Minimum Degree Heuristic and the Minimal Triangulation Process
}

\author{
Anne Berry $^{*} \quad$ Pinar Heggernes ${ }^{\dagger} \quad$ Geneviève Simonet ${ }^{\ddagger}$
}

\begin{abstract}
The Minimum Degree Algorithm, one of the classical algorithms of sparse matrix computations, is a heuristic for computing a minimum triangulation of a graph. It is widely used as a component in every sparse matrix package, and it is known to produce triangulations with few fill edges in practice, although no theoretical bound or guarantee has been shown concerning the amount of fill it introduces. An additional remarkable property of Minimum Degree observed in practice is that it often produces a minimal triangulation. Despite extensive research on optimizing the running time of this heuristic, few theoretical results are known about it.

Our goal in this paper is to examine the theoretical reasons behind such good behaviour. We give new invariants which explain, at least in part, the mechanisms underlying this heuristic. We show that Minimum Degree is in fact robust, in the sense that it is resilient to error, as even when an undesirable triangulating edge with respect to minimal triangulation is added at some step of the algorithm, at later steps the chances of adding only desirable edges remain intact. We also use our new graph theoretical insight to propose an improvement of this heuristic, which introduces at most as many fill edges as Minimum Degree but is guaranteed to yield a minimal triangulation.
\end{abstract}

\section{Introduction}

For the past forty years, problems arising from applications have given rise to challenges for graph theorists, and thus also to a wealth of graph-theoretic results.

One of these is computing a minimum triangulation. Though the problem originally arised from the field of sparse matrix computation [10,24], it has applications in various areas of computer science, such as database management systems [26], knowledge based systems [15] and computer vision [6].

Large sparse symmetric systems of equations arise in many areas of engineering,as the structural analysis of a car body, or the modeling of air flow around an airplane wing. The physical structure can often be thought of as covered by a mesh where each point is connected to a few other points, and the related sparse matrix can simply be regarded as an adjacency matrix of this mesh. Such systems are solved through standard methods of linear algebra, like Gaussian elimination, and during this process non-zero entries are inserted into places that originally held zeros, which increases both the storage requirement and the time needed to solve the system. It was observed very early that finding a good pivotal ordering of this matrix can reduce the amount of fill thus introduced: in 1957, Markowitz [18] introduced the idea behind the algorithm known today as Minimum Degree, choosing a pivot row and column at each step of the Gaussian elimination to decrease the product of the number of corresponding off-diagonal non-zeros. Tinney and Walker [27] later applied this idea to symmetric matrices, and Rose [24] developed a graph theoretical model of it.

\footnotetext{
${ }^{*}$ LIMOS UMR CNRS 6158, Bat Isima, BP 10 125, 63173 Aubière, France. berry@isima.fr

$\dagger$ Department of Informatics, University of Bergen, N-5020 Bergen, Norway. pinar@ii.uib.no

$\ddagger$ LIRMM, 161 Rue Ada, 34392 Montpellier, France. simonet@lirmm.fr
} 
It appeared rapidly that solving such systems is easy when the undirected graph represented by the corresponding matrix is chordal (or triangulated). This is due to the property that finding a good pivotal ordering on the matrix is equivalent to finding in the corresponding underlying graph a perfect elimination scheme [13], which means repeatedly finding a simplicial vertex and removing it. This process was shown by Fulkerson and Gross [9] in 1965 to characterize chordal graphs.

When the underlying graph fails to be chordal, however, a solution can be found by embedding the graph into a chordal graph by adding edges, a process called triangulation.

As early as 1961, Parter [22] presented an algorithm, known as Elimination Game (EG), which simulates Gaussian elimination by repeatedly choosing a vertex and adding edges to make its neighborhood into a clique before removing it, thus introducing the connection between sparse matrices and graphs. In view of the results in [9], no non-zero entries are introduced when the underlying graph is chordal, and when this fails to be the case, EG produces a triangulation of the input graph.

As can be observed on the example in Figure 1, the number of fill edges in the resulting triangulation is heavily dependent on the order in which EG processes the vertices.

As mentioned above, it is of primary importance to add as few edges as possible when running Elimination Game. The corresponding problem is that of computing a minimum triangulation, which unfortunately is NP-complete, as shown by Yannakakis [28].

Although it is possible to compute in polynomial time a triangulation which is minimal (meaning that an inclusion-minimal set of edges is added, [20], [25]), this process is not pursued by the sparse matrix society in practice, since such a triangulation can be far from minimum, as can be seen from the example of Figure 1(b).

As a result, researchers have resorted to heuristics, of which one of the most universally used and studied is Minimum Degree, or MD: this runs the Elimination Game by choosing at each step a vertex of minimum degree in the transitory elimination graph, as illustrated by Figure 1(d). This algorithm is widely used in practice, and it is known to produce low fill triangulations. In addition, MD is also observed [5] to produce triangulations which are often minimal or close to minimal.

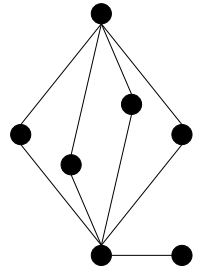

(a)

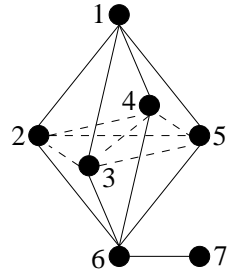

(b)

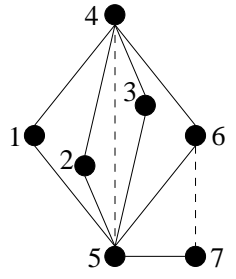

(c)

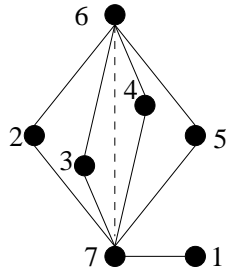

(d)

Figure 1: (a) A graph $G$, and various triangulations of $G$ by EG through the given orderings: (b) A minimal triangulation with $O\left(n^{2}\right)$ fill edges. (c) A non-minimal triangulation of $G$ with less fill. (d) A minimum triangulation of $G$.

MD has given rise to a large amount of research with respect to improving the running time of its practical implementations, and the number of papers written on this subject is in the hundreds $[1,11]$. However, very little is known theoretically as to its quality. It has in fact been analyzed theoretically only to a limited extent, which makes it difficult to gain control over this heuristic in order to improve it yet further, although recent research has been done on algorithms for low fill minimal triangulations $[5,7,23]$.

In this paper, we use recent graph theoretical results on minimal triangulation and minimal separation to explain, at least in part, why MD yields such good results. In fact, it turns out that one of the reasons why MD works so well is that the EG algorithm is remarkably robust, in the sense that it is resilient to error: if at some step of the process an undesirable edge with respect to 
minimal triangulation is added, at later steps the chances of adding only desirable edges remain intact. One of our contributions here is that we use the insight we have gained on the mechanisms which govern EG and MD to propose an algorithmic process which improves the results obtained by MD, giving triangulations that are both minimal and have low fill.

The remainder of this work is organized as follows: Section 2 gives the graph theoretic background, introduces EG formally, and gives previous results on minimal separation and minimal triangulation. In Section 3, we give some new invariants for EG and MD, and explain why these algorithms are resilient to error, and why in many cases MD computes a minimal triangulation. Section 4 proposes a new algorithmic process in view of using the results of the paper to improve MD.

\section{Preliminaries}

Given a graph $G=(V, E)$, we denote $n=|V|$ and $m=|E|$. For any subset $A$ of $V, G(A)$ denotes the subgraph of $G$ induced by $A$. For the sake of simplicity, will use informal notations such as $H=G+\{e\}+\{x\}$ when $H$ is obtained from $G$ by adding edge $e$ and vertex $x$. For any vertex $v$ of $G, N_{G}(v)$ denotes the neighborhood of $v$ in $G$, and $N_{G}[v]$ denotes the set $N_{G}(v) \cup\{v\}$. For a given set of vertices $X \subset V, N_{G}(X)=\cup_{v \in X} N_{G}(v)-X$, and $N_{G}[X]=\cup_{v \in X} N_{G}(v) \cup X$ (we will omit the subscripts when there is no ambiguity).

A clique is a set of pairwise adjacent vertices. A vertex is simplicial if its neighborhood is a clique. We will say that we saturate a set of vertices $X$ when we add to the graph all the edges necessary to make $X$ into a clique. A graph is chordal, or triangulated, if it contains no chordless cycle of length $\geq 4$. The set $F$ of edges added to an arbitrary graph $G=(V, E)$ to obtain a triangulation $H=(V, E+F)$ of $G$ is called a fill.

$(G, \alpha)$ will denote a graph $G$, the vertices of which are ordered according to $\alpha$. We will use $\alpha=\left(v_{1}, v_{2}, \ldots, v_{n}\right)$, where $\alpha\left(v_{i}\right)=i$.

The algorithmic description of Elimination Game (EG) given below defines the notations we will use in the rest of this paper:

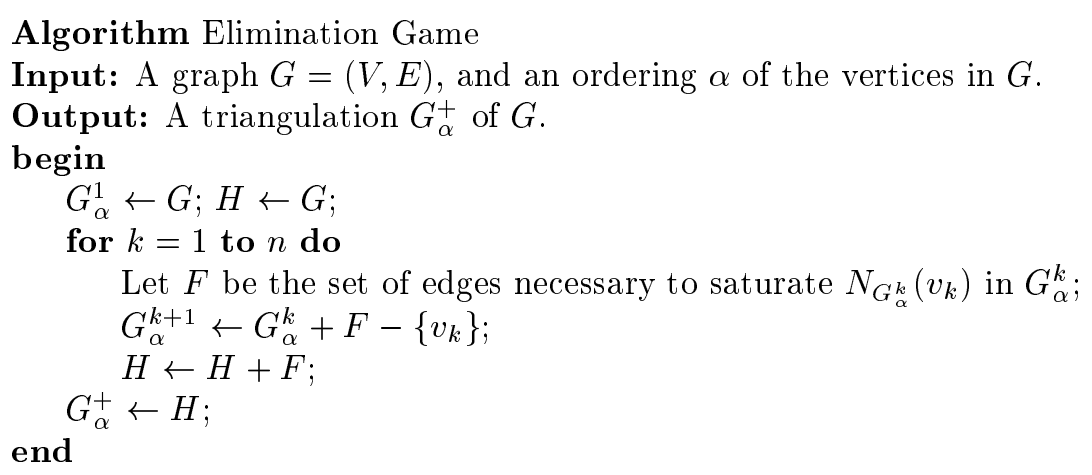

According to the definition used in [20], we will call $G_{\alpha}^{k}\left(\left\{v_{k}, \ldots, v_{n}\right\}-N_{G_{\alpha}^{k}}\left[v_{k}\right]\right)$ the section graph at step $k$. The edges added during an execution of EG are well defined by the following lemma by Rose, Tarjan and Lueker:

Lemma 2.1 ([25]) Given $(G, \alpha), v_{i} v_{j}$ is an edge of $G_{\alpha}^{+}$if and only if $v_{i} v_{j}$ is an edge of $G$ or there is a path in $G$ between $v_{i}$ and $v_{j}$, all intermediate vertices of which have a number which is strictly smaller than $\min \{i, j\}$.

The Minimum Degree (MD) heuristic is based on EG: it takes as input a graph $G$, and computes an ordering $\alpha$ along with the corresponding triangulation $G_{\alpha}^{+}$, by choosing at each step a vertex of minimum degree in $G_{\alpha}^{k}$ and numbering it as $v_{k}$. 
Before proceeding to the next section, we will need some results on minimal separation. The notion of a minimal separator was introduced by Dirac [8] to characterize chordal graphs. Given a graph $G=(V, E)$, a vertex set $S \subset V$ is a minimal separator if $G(V-S)$ has at least two connected components $C_{1}$ and $C_{2}$ such that $N_{G}\left(C_{1}\right)=N_{G}\left(C_{2}\right)=S\left(C_{1}\right.$ and $C_{2}$ are called full components $)$.

Characterization 2.2 ([8]) A graph is chordal iff all its minimal separators are cliques.

Recent research $[14,21,2]$ has shown that minimal separators are central to minimal triangulations. The idea behind this is that forcing a graph into respecting Dirac's characterization will result into a minimal triangulation, by repeatedly choosing a not yet processed minimal separator and saturating it. We will need the definition of crossing separators, which characterize the separators which disappear when a saturation step of this process is executed:

Definition 2.3 ([14]) Let $S$ and $S^{\prime}$ be two minimal separators of $G$. $S$ and $S^{\prime}$ are said to be crossing if there exist two connected components $C_{1}, C_{2}$ of $G(V-S)$, such that $S^{\prime} \cap C_{1} \neq \emptyset$ and $S^{\prime} \cap C_{2} \neq \emptyset$ (the crossing relation is symmetric).

The saturation process described above can be generalized by choosing and simultaneously saturating a set of pairwise non-crossing minimal separators instead of a single minimal separator at each step, until a chordal graph is obtained. We will refer to this generalized process as the Saturation Algorithm. Given a set $\mathcal{S}$ of minimal separators of $G$, we will denote $G_{\mathcal{S}}$ the graph obtained from $G$ by saturating all the separators belonging to $\mathcal{S}$.

The following results from the works of Kloks, Kratsch and Spinrad [14] and Parra and Scheffler [21] provide a proof of this algorithm and will be used in Sections 3 and 4 .

Theorem 2.4 ([21]) A graph $H=(V, E+F)$ is a minimal triangulation of $G=(V, E)$ iff there is a maximal set $\mathcal{S}$ of pairwise non-crossing minimal separators of $G$ such that $H=G_{\mathcal{S}}$.

Corollary 2.5 A graph $H=(V, E+F)$ is a minimal triangulation of $G=(V, E)$ iff $H$ is chordal and there is a set $\mathcal{S}$ of pairwise non-crossing minimal separators of $G$ such that $H=G_{\mathcal{S}}$.

We will also need the following Lemmas, which follow from the work in [21]:

Lemma 2.6 ([21]) Let $G=(V, E)$ be a graph, let $\mathcal{S}$ and $\mathcal{S}^{\prime}$ be sets of pairwise non-crossing minimal separators of $G$ and $G_{\mathcal{S}}$, respectively. Then $\mathcal{S} \cup \mathcal{S}^{\prime}$ is a set of pairwise non-crossing minimal separators of $G$.

Lemma 2.7 ([21]) Let $G=(V, E)$, be a graph and $\mathcal{S}$ a set of pairwise non-crossing minimal separators of $G$. Then any minimal triangulation of $G_{\mathcal{S}}$ is a minimal triangulation of $G$.

We will also use the notion of substar, which was introduced by Lekkerkerker and Boland [16] in connection with their characterization of chordal graphs.

Definition 2.8 ([16]) Given a graph $G=(V, E)$ and a vertex $x$ of $G$, the substars of $x$ in $G$ are the neighborhoods in $G$ of the connected components of $G(V-N[x])$.

In fact, although Lekkerkerker and Boland seemed not to be aware of this, the set of substars of some vertex $x$ is exactly the set of minimal separators included in the neighborhood of $x$. LBsimpliciality of a vertex was defined in [3] in the following way for more convenient terminology.

Definition 2.9 A vertex $x$ is LB-simplicial if every substar of $x$ is a clique.

This was implicitly used by [16] to characterize chordal graphs as graphs such that every vertex is LB-simplicial, but the notion of substar is also very useful in the context of minimal triangulation, because it provides a fast and easy way of repeatedly finding sets of pairwise non-crossing minimal separators when running the Saturation Algorithm. This is fully described in [4], with in particular the following lemma:

Lemma 2.10 ([4]) The substars of a vertex $x$ in a graph $G$ are pairwise non-crossing in $G$. 


\section{Properties of Elimination Game related to minimal trian- gulation}

We will now examine how EG behaves with respect to the minimal separators of the graph which is to be triangulated.

\subsection{EG and partial minimal triangulation}

We will first extend the definition of substar given in Section 2 to that of substars of $(G, \alpha)$.

Definition 3.1 Given $(G=(V, E), \alpha)$, we will say that a set $S \subset V$ of vertices is a substar of $(G, \alpha)$ if there is some step $k$ of $E G$ such that $S$ is a substar of $v_{k}$ in $G_{\alpha}^{k}$, which will be referred to as $a$ substar defined at step $k$ of $E G$.

Clearly, during the execution of the EG, at each step $k$, making the currently processed vertex $v_{k}$ simplicial will saturate these substars, and may also add some extraneous edges which do not have both endpoints in some common substar, so that two kinds of edges can be added:

- Edges which have both endpoints in some common substar defined at step $k$. We will refer to these edges as substar fill edges.

- Edges which do not have both endpoints in some common substar defined at step $k$. We will refer to these as extraneous edges.

In Section 2, we mentioned that in $G$ and for a given vertex $v$, the substars of $v$ are the minimal separators included in the neighborhood of $v$. One of our most interesting discoveries is that, in fact, all the substars defined by EG are minimal separators of the input graph, whether or not extraneous edges have been added at earlier steps. This fact is stated in Theorem 3.3, and its proof is based on the following Lemma, which is interesting in its own right, as it describes a strong correspondence between the structures of $G$ and $G_{\alpha}^{k}$.

Lemma 3.2 Given $(G=(V, E), \alpha)$ and an integer $k \in[1, n]$, let $G_{\alpha}^{k}=\left(V_{\alpha}^{k}, E_{\alpha}^{k}\right)$ and $S \subseteq V_{\alpha}^{k}$. The connected components of $G_{\alpha}^{k}\left(V_{\alpha}^{k}-S\right)$ are the sets $C \cap V_{\alpha}^{k}$ where $C$ is a connected component of $G(V-S)$ such that $C \cap V_{\alpha}^{k} \neq \emptyset$, with the same neighborhoods, i.e. $N_{G_{\alpha}^{k}}\left(C \cap V_{\alpha}^{k}\right)=N_{G}(C)$.

The proof of this lemma is given in the Appendix.

Theorem 3.3 Every substar of $(G, \alpha)$ is a minimal separator of $G$.

Proof : Let $S$ be a substar defined at step $k . S$ is a minimal separator of $G_{\alpha}^{k}$; by Lemma 3.2, there are at least as many full components of $G(V-S)$ as of $G_{\alpha}^{k}\left(V_{\alpha}^{k}-S\right)$. So $S$ is also a minimal separator of $G$.

We are now ready to state our Main Theorem:

Main Theorem 3.4 The set of substars of $(G, \alpha)$ forms a set of pairwise non-crossing minimal separators of $G$.

Proof : Let $S$ and $S^{\prime}$ be two substars of $(G, \alpha)$ defined at steps $k$ and $k^{\prime}$ respectively, with $k \leq k^{\prime}$. By Theorem 3.3, they are both minimal separators of $G$. Let us show that they are non-crossing in $G$. If $k=k^{\prime}$ then they are non-crossing in $G_{\alpha}^{k}$ by Lemma 2.10 , so they are non-crossing in $G$ by Lemma 3.2. We suppose now that $k<k^{\prime}$. $S$ is a clique of $G_{\alpha}^{k+1}$ and $S^{\prime} \subseteq V_{\alpha}^{k+1}$, so there is a connected component $C$ of $G_{\alpha}^{k+1}\left(V_{\alpha}^{k+1}-S^{\prime}\right)$ such that $S \subseteq S^{\prime} \cup C$. By Lemma 3.2, there is a connected component $C^{\prime}$ of $G\left(V-S^{\prime}\right)$ containing $C$, so $S \subseteq S^{\prime} \cup C^{\prime}$. Hence $S$ and $S^{\prime}$ are non-crossing in $G$. 


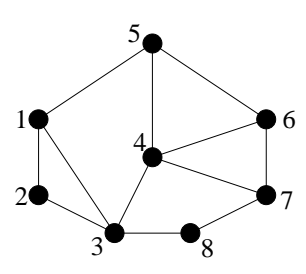

(a)

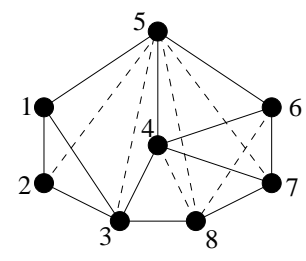

(b)

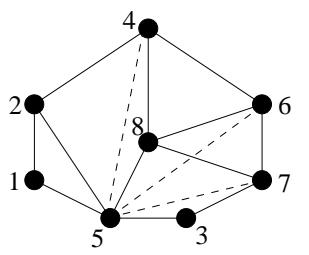

(c)

Figure 2: Two executions of EG on the same graph with (b) an arbitrary ordering, and (c) an MD ordering.

Note that this theorem does not guarantee that the set of substars defines a set of pairwise non-crossing minimal separators which is maximal. For instance, for any non complete graph $G$, if $v_{1}$ is a universal vertex of $G$ then there is no substar of $(G, \alpha)$ whereas $G$ has at least one minimal separator. A less trivial counterexample is given in Figure 2(b) of Example 3.5.

Example 3.5 Figure 2 shows two executions of EG on graph $G$.

(a) A graph $G$ and an ordering $\alpha$ are given. The minimal separators of $G$ are: $\{1,3\},\{3,5\}$, $\{3,7\},\{1,4,6\},\{1,4,7\},\{1,4,8\},\{3,5,7\},\{4,5,7\},\{4,5,8\},\{4,6,8\},\{3,4,6\}$.

(b) We now demonstrate the execution of $E G$ on $(G, \alpha)$ resulting in the graph shown in (b). Step 1: $N(1)=\{2,3,5\}, C_{1}=\{4,6,7,8\}, N\left(C_{1}\right)=\{3,5\}$; substar fill edge 35 and extraneous edge 25 are added. Step 2: $N(2)=\{3,5\} ; C_{2}=\{4,6,7,8\}, N\left(C_{2}\right)=\{3,5\} ; 2$ is simplicial, so no edge is added. Step 3: $N(3)=\{4,5,8\}, C_{3}=\{6,7\}, N\left(C_{3}\right)=\{4,5,8\}$; substar fill edges 48 and 58 are added. Step 4: $N(4)=\{5,6,7,8\} ; 4$ is universal, so no component is defined; extraneous edges 57 and 68 are added; the remaining graph becomes a clique; no further edge is added.

The set of substars of $(G, \alpha)$ is thus $\{\{3,5\},\{4,5,8\}\}$, which is a set of pairwise non-crossing minimal separators of $G$, but not a maximal one as $\{\{3,5\},\{4,5,8\},\{4,5,7\}\}$ and $\{\{3,5\},\{4,5,8\}$, $\{4,6,8\}\}$ are also sets of pairwise non-crossing minimal separators of $G$. If only substar fill edges are preserved, a chordless cycle 5678 remains in the graph thus obtained. In order to saturate a maximal set of pairwise non-crossing minimal separators of $G,\{4,5,7\}$ or $\{4,6,8\}$ should also be saturated.

(c) On the same graph, MD yields a minimal (and even minimum) triangulation.

We would like to end this subsection with a discussion on the robustness of EG and MD regarding the process of defining non-crossing minimal separators of $G$. If, during the EG process, no extraneous edge is added, then the triangulation which is computed is minimal. However, due to Theorem 3.4, even when extraneous edges have been added, all substar fill edges added later belong to a set of pairwise non-crossing minimal separators of $G$, and therefore to a minimal triangulation of $G$. Thus if only a few extraneous edges are added during EG process, they will not destroy the property that all the substar fill edges are "useful" edges and that these few extraneous edges are the only "unnecessary" edges introduced. This makes EG a fault-tolerant procedure.

\subsection{Conditions for EG and MD to produce minimal triangulations}

We have seen that EG does not necessarily compute a minimal triangulation. However, as mentioned earlier, MD is observed in practice to often produce orderings that are minimal or close to minimal, in addition to low fill. We will now give an explanation of this good behaviour of MD through Lemma 3.6.

Lemma 3.6 Let $v_{k}$ be a vertex of minimum degree in $G_{\alpha}^{k}$ such that the union of all substars defined at step $k$ is equal to a substar $S$ defined at step $k$. Then only substar fill edges are added at step $k$. 
Proof : Assume by contradiction that there is an extraneous edge $y z$ added at step $k$. Then $y$ or $z$, say $y$, is not in $S$. $N_{G_{\alpha}^{k}}(y) \subseteq N_{G_{\alpha}^{k}}\left[v_{k}\right]-\{y, z\}$, so that $\left|N_{G_{\alpha}^{k}}(y)\right|<\left|N_{G_{\alpha}^{k}}\left(v_{k}\right)\right|$, which contradicts the fact that $v_{k}$ is a vertex of minimum degree in $G_{\alpha}^{k}$.

This result shows in particular that when the section graph is connected at some step of MD, then fill edges are added at that step only within the single substar defined. As is clear from the proof of this lemma, this is not the case in general for EG. Moreover, in most practical applications [19], the input graph is sparse; although no statistical result has been established on this, intuitively, a vertex $x$ of minimum degree quite often defines only one substar, which usually corresponds to a connected section graph. MD run on sparse graphs thus stands a significantly higher chance of generating minimal triangulations than EG.

\section{Improving the results of Minimum Degree}

Based on the results of the previous sections, we now present a variant of MD that produces a minimal triangulation and yields at most the same amount of fill, because it computes a minimal triangulation which is a subgraph of that computed by MD.

We have seen in Section 3 that the set $\mathcal{S}$ of non-crossing minimal separators of $G$ defined by the set of all substars of $(G, \alpha)$ is not necessarily maximal. Thus if we remove all extraneous edges from $G_{\alpha}^{+}$(or equivalently, add to $G$ only the substar fill edges), the graph $G_{\S}$ thus obtained might fail to be triangulated. However, by Lemma 2.7, we know that any minimal triangulation $H$ of $G_{\delta}$ is a minimal triangulation of $G$. We thus propose a process which computes a minimal triangulation of the graph $G_{\S}$ obtained by running $\mathrm{MD}$ on $G$ and removing the extraneous edges. In order to give the MD approach its chances, we will repeat the process of running MD on the partially triangulated graph obtained and removing the extraneous edges, until a chordal graph is obtained. However, at the beginning of each iteration, we will remove all LB-simplicial vertices, according to the following result:

Lemma 4.1 Let $G=(V, E)$ be a graph, let $X$ be the set of LB-simplicial vertices of $G$, let $G^{\prime}=G(V-X)=\left(V^{\prime}, E^{\prime}\right)$; then for any minimal triangulation $H^{\prime}=\left(V^{\prime}, E^{\prime}+F^{\prime}\right)$ of $G^{\prime}$, the graph $H=\left(V, E+F^{\prime}\right)$ is a a minimal triangulation of $G$.

Proof : $\quad H$ is chordal because for any cycle $C$ in $H$ of length $\geq 4$, either $C$ is in $H^{\prime}$ and then $C$ has at least one chord, or $C$ contains a vertex $x$ of $X$ and then the neighbors of $x$ in $C$ induce a chord of $C$, as they belong to the substar of $x$ defined by the component containing the other vertices of $C$. So $H$ is a a triangulation of $G$. It is a minimal one because for any chordal graph $H_{1}=\left(V, E+F_{1}^{\prime}\right)$ with $F_{1}^{\prime} \subseteq F^{\prime}$, the graph $H_{1}^{\prime}=\left(V^{\prime}, E^{\prime}+F_{1}^{\prime}\right)$ is chordal too, so that $F_{1}^{\prime}=F^{\prime}$.

Thus the LB-simplicial vertices can only cause MD to add extraneous edges, as well as unnecessarily increasing some vertex degrees, which justifies our systematically eliminating them from the graph at each step. Note that any step of the MD process tends to create LB-simplicial vertices, so removing these can make a significant difference regarding the quality of the fill obtained.

We now present the new algorithm.

Algorithm Minimal Minimum Degree (MMD)

Input: A graph $G$.

Output: A minimal triangulation $H$ of $G$.

begin

Run MD on $G$, which defines an ordering $\alpha$ and a set of substars $\mathcal{S}$ of $(G, \alpha)$;

$G^{\prime} \leftarrow G_{S} ; H \leftarrow G_{S} ;$

while $G^{\prime}$ is not chordal do

Remove all LB-simplicial vertices from $G^{\prime}$;

Run MD on $G^{\prime}$, which defines an ordering $\alpha$ and a set of substars $\mathcal{S}$ of $\left(G^{\prime}, \alpha\right)$; 
end $\quad G^{\prime} \leftarrow G_{\delta}^{\prime} ; H \leftarrow H_{\delta}$

It should be noted that graphs can be constructed such that no execution of MD can produce a minimal triangulation. Such an example is a graph consisting of two large cliques, connected by a single path of length $\geq 2$. The graph is chordal, but vertices on the path will be chosen by MD at first steps, introducing unnecessary fill. MMD will not encounter any problem with that kind of graph, since the only minimal triangulation of a chordal graph is the graph itself.

We now prove that MMD gives a fill which is as least as good a that of MD, by showing that MMD yields a subgraph of $G_{\alpha}^{+}$.

Theorem 4.2 Let $\mathcal{S}$ be the set of substars of $(G, \alpha)$. Then any minimal triangulation $H$ of $G_{\S}$ is a minimal triangulation of $G$ which is a subgraph of $G_{\alpha}^{+}$.

The proof of this theorem is given in the Appendix.

Theorem 4.3 Minimal Minimum Degree computes a minimal triangulation of $G$ which is a subgraph of $G_{\alpha}^{+}$, where $\alpha$ is the $M D$ ordering computed at the beginning of the algorithm.

Proof : MMD terminates, because at the beginning of each step, the LB-simplicial vertices are all removed, so that the vertex of minimum degree which is chosen first is not LB-simplicial; as a result, making it simplicial cannot fail to add at least one substar fill edge. Let us prove MMD correctness. Let $\mathrm{H}$ be the output graph, let $\mathcal{S}_{0}$ be the set of substars computed at the beginning of the algorithm and $\mathcal{S}^{\prime}$ be the union of those computed in the while-loop. Thus $H=\left(G_{\mathcal{S}_{0}}\right)_{\mathcal{S}^{\prime}}$, $G_{\mathcal{S}_{0}}$ being the input graph of the while-loop. By Theorem 4.2 it is sufficient to show that $H$ is a minimal triangulation of $G_{\delta_{0}}$, or more generally that for any input graph $G^{\prime}$ of the while-loop, the graph $G_{\mathcal{S}^{\prime}}^{\prime}$, where $\mathcal{S}^{\prime}$ is the union of the sets of substars computed in the while-loop, is a minimal triangulation of $G^{\prime}$. Let us prove this property by induction on the number $p$ of iterations of the while-loop before $G^{\prime}$ gets chordal. It trivially holds for $p=0$, as in that case $G^{\prime}$ is chordal and $\mathcal{S}^{\prime}$ is the empty set. We suppose that it holds when the number of iterations of the while-loop before $G^{\prime}$ gets chordal is $p$. Let us show that it holds when this number is $p+1$. Let $G_{1}^{\prime}$ be the graph obtained from $G^{\prime}$ by removing all its LB-simplicial vertices, let $\mathcal{S}_{1}^{\prime}$ be the set of substars computed at the first iteration of the while-loop and $\mathcal{S}^{\prime \prime}$ be the union of those computed at the following iterations, and let $G^{\prime \prime}$ be the graph obtained at the end of the first iteration. Thus $G^{\prime \prime}=\left(G_{1}^{\prime}\right)_{\mathcal{S}_{1}^{\prime}}$ and $\mathcal{S}^{\prime}=\mathcal{S}_{1}^{\prime} \cup \mathcal{S}^{\prime \prime}$. By induction hypothesis, $G_{\mathcal{S}^{\prime \prime}}^{\prime \prime}$ is a minimal triangulation of $G^{\prime \prime}$, so by Theorem 3.4 and Lemma 2.7 , it is also a minimal triangulation of $G_{1}^{\prime} . G_{\mathcal{S}^{\prime \prime}}^{\prime \prime}=\left(\left(G_{1}^{\prime}\right)_{\mathcal{S}_{1}^{\prime}}\right)_{\mathcal{S}^{\prime \prime}}=\left(G_{1}^{\prime}\right)_{\mathcal{S}_{1}^{\prime} \cup \mathcal{S}^{\prime \prime}}=\left(G_{1}^{\prime}\right)_{\mathcal{S}^{\prime}}$. Thus the graphs $G_{\mathcal{S}^{\prime}}^{\prime}$ and $G_{\mathcal{S}^{\prime \prime}}^{\prime \prime}$ are obtained from $G^{\prime}$ and $G_{1}^{\prime}$ respectively by adding the same set $F^{\prime}$ of edges, so by Lemma $4.1, G_{\delta^{\prime}}^{\prime}$ is a minimal triangulation of $G^{\prime}$, which completes the proof by induction and therefore the proof of MMD correctness.

With practical tests, we have compared MMD against MD, with respect to the number of edges in the resulting triangulation. We have done a simple and straightforward implementation of MMD in Matlab, and we have run the tests both on randomly generated graphs of varying density, and on graphs from Matrix Market [19]. On each graph $G$, we first generated an MD ordering $\alpha$. Then we compared the number of fill edges in $G_{\alpha}^{+}$to the number of fill edges produced by MMD. As expected by Theorem 4.3, the number of fill edges resulting from MMD was always less than or equal to the number of fill edges resulting from MD. An interesting point is that on most graphs, MMD required only two iterations of the while-loop. The reduction in the number of fill edges achieved by MMD was not very large, because of MD's remarkably good performances. However, this improved algorithm may both give significant results on very large graphs and help researchers gain a better evaluation of how close MD gets to an optimal solution.

Finally, we would like to mention that existing MD codes in use are very fast although the theoretical running time of these implementations is not good [12]. In addition, other iterative procedures for computing minimal triangulations have been implemented to run fast in practice [23]. Thus we believe that, with some effort, MMD can be implemented to run efficiently in practice. 


\section{Conclusion}

Our contributions in this paper are threefold. We have found new invariants for the Elimination Game process, proving that it defines and saturates a set of minimal separators of the input graph, with a remarkably fault-tolerant behavior. We have shown that Minimum Degree has additional properties that gives it a high chance of actually producing minimal triangulations, thereby explaining this practical behavior of MD. Finally, we have given a new algorithm that produces minimal triangulations with low fill, based on our findings about EG and MD.

\section{Acknowledgment}

The authors thank Elias Dahlhaus for interesting discussions on minimal triangulations.

\section{References}

[1] P. Amestoy, T. A. Davis, and I. S. Duff. An approximate minimum degree ordering algorithm. SIAM J. Matrix Anal. Appl., 17:886-905, 1996.

860-861, Jan. 1999.

[2] A. Berry. Désarticulation d'un graphe. PhD Dissertation, LIRMM, Montpellier, December 1998.

[3] A. Berry, J. R. S. Blair, and P. Heggernes. Maximum Cardinality Search for Computing Minimal Triangulations. In L. Kucera, editor, Graph Theoretical Concepts in Computer Science WG 2002, Springer Verlag, 2002. Lecture Notes in Computer Science.

[4] A. Berry, J.-P. Bordat, P. Heggernes, G. Simonet, and Y. Villanger. A wide-range algorithm for minimal triangulation from an arbitrary ordering. Reports in Informatics 243, University of Bergen, Norway, 2003, and Research Report 02-200, LIRRM, Montpellier, France. Submitted to Journal of Algorithms, November 2002.

[5] J. R. S. Blair, P. Heggernes, and J. A. Telle. A practical algorithm for making filled graphs minimal. Theoretical Computer Science, 250:124-141,2001.

[6] F. R. K. Chung and D. Mumford. Chordal completions of planar graphs. J. Comb. Theory, 31:96-106, 1994.

[7] E. Dahlhaus. Minimal elimination ordering inside a given chordal graph. In R. H. Möhring, editor, Graph Theoretical Concepts in Computer Science, pages 132-143. Springer Verlag, 1997. Lecture Notes in Computer Science 1335.

[8] G.A. Dirac. On rigid circuit graphs. Anh. Math. Sem. Univ. Hamburg, 25:71-76, 1961.

[9] D. R. Fulkerson and O. A. Gross. Incidence matrices and interval graphs. Pacific Journal of Math., 15:835-855, 1965.

[10] J. A. George and J. W. H. Liu. Computer Solution of Large Sparse Positive Definite Systems. Prentice-Hall Inc., Englewood Cliffs, New Jersey, 1981.

[11] J. A. George and J. W. H. Liu. The evolution of the minimum degree ordering algorithm. SIAM Review, 31:1-19, 1989.

[12] P. Heggernes, S. Eisenstat, G. Kumfert, and A. Pothen. The computational complexity of the Minimum Degree algorithm. Proceedings of 14th Norwegian Computer Science Conference, NIK 2001, University of Troms, Norway. Also available as ICASE Report 2001-42, NASA/CR2001-211421, NASA Langley Research Center, USA. 
[13] M.C. Golumbic. Algorithmic Graph Theory and Perfect Graphs. Academic Press, New York, 1980 .

[14] T. Kloks, D. Kratsch, and J. Spinrad. On treewidth and minimum fill-in of asteroidal triplefree graphs. Theoretical Computer Science, 175:309-335, 1997.

[15] S. L. Lauritzen and D. J. Spiegelhalter. Local computations with probabilities on graphical structures and their applications to expert systems. J. Royal Statist. Soc., ser B, 50:157-224, 1988.

[16] C. G. Lekkerkerker and J. Ch. Boland. Representation of a finite graph by a set of intervals on the real line. Fund. Math., 51:45-64, 1962.

[17] J. W. H. Liu. Equivalent sparse matrix reorderings by elimination tree rotations. SIAM J. Sci. Stat. Comput., 9:424-444, 1988.

[18] H. M. Markowitz. The elimination form of the inverse and its application to linear programming. Management Science, 3:255-269, 1957.

[19] Matrix Market Web site: http://math.nist.gov/MatrixMarket/

[20] T. Ohtsuki, L. K. Cheung, and T. Fujisawa. Minimal triangulation of a graph and optimal pivoting order in a sparse matrix. J. Math. Anal. Appl., 54:622-633, 1976.

[21] A. Parra and P. Scheffler. How to use the minimal separators of a graph for its chordal triangulation. Proceedings of the 22nd International Colloquium on Automata, Languages and Programming (ICALP '95), Lecture Notes in Computer Science, 944:123-134, 1995.

[22] S. Parter. The use of linear graphs in Gauss elimination. SIAM Review, 3:119-130, 1961.

[23] B. Peyton. Minimal orderings revisited. SIAM J. Matrix Anal. Appl., 23:271-294, 2001.

[24] D. J. Rose. A graph-theoretic study of the numerical solution of sparse positive definite systems of linear equations. In R. C. Read, editor, Graph Theory and Computing, pages 183-217. Academic Press, 1972.

[25] D. J. Rose, R. E. Tarjan, and G. S. Lueker. Algorithmic aspects of vertex elimination on graphs. SIAM J. Comput., 5:266-283, 1976.

[26] R. E. Tarjan and M. Yannakakis. Simple linear-time algorithms to test chordality of graphs, test acyclicity of hypergraphs, and selectively reduce acyclic hypergraphs. SIAM J. Comput., 13:566-579, 1984.

[27] W. F. Tinney and J. W. Walker. Direct solutions of sparse network equations by optimally ordered triangular factorization In Proceedings of the IEEE, 55:1801-1809, 1967.

[28] M. Yannakakis. Computing the minimum fill-in is NP-complete. SIAM J. Alg. Disc. Meth., $2: 77-79,1981$. 


\section{Appendix}

In the following proofs, for any graph $G=(V, E)$ and $S \subseteq V, \mathcal{C}_{G}(S)$ denotes the set of connected components of $G(V-S)$. We will use the following characterization (C) of the edges of $G_{\alpha}^{k}$, with $G_{\alpha}^{k}=\left(V_{\alpha}^{k}, E_{\alpha}^{k}\right)$ and $V_{\alpha}^{k}=\left\{v_{k}, v_{k+1}, \ldots, v_{n}\right\}$ :

(C): for any distinct $i, j \in[k, n], v_{i} v_{j} \in E_{\alpha}^{k}$ iff there is a path in $G$ between $v_{i}$ and $v_{j}$ (the path may have only one edge) all intermediate vertices of which are numbered $<k$ (i.e. do not belong to $\left.V_{\alpha}^{k}\right)$.

The proof of $(\mathbf{C})$ is similar to that of Lemma 2.1.

Lemma 3.2: Given $(G=(V, E), \alpha)$ and an integer $k \in[1, n]$, let $G_{\alpha}^{k}=\left(V_{\alpha}^{k}, E_{\alpha}^{k}\right)$ and $S \subseteq V_{\alpha}^{k}$. The connected components of $G_{\alpha}^{k}\left(V_{\alpha}^{k}-S\right)$ are the sets $C \cap V_{\alpha}^{k}$ where $C$ is a connected component of $G(V-S)$ such that $C \cap V_{\alpha}^{k} \neq \emptyset$, with the same neighborhoods, i.e. $N_{G_{\alpha}^{k}}\left(C \cap V_{\alpha}^{k}\right)=N_{G}(C)$.

Proof : Let $S \subseteq V_{\alpha}^{k}$. We have to prove that $\mathcal{C}_{G_{\alpha}^{k}}(S)=\left\{C \cap V_{\alpha}^{k}, C \in \mathcal{C}_{G}(S) \mid C \cap V_{\alpha}^{k} \neq \emptyset\right\}$ and $\forall C \in \mathcal{C}_{G}(S), N_{G_{\alpha}^{k}}\left(C \cap V_{\alpha}^{k}\right)=N_{G}(C)$. Let $C \in \mathcal{C}_{G}(S)$ such that $C \cap V_{\alpha}^{k} \neq \emptyset$ and let $C^{\prime}=C \cap V_{\alpha}^{k}$. Let us show that $C^{\prime} \in \mathcal{C}_{G_{\alpha}^{k}}(S)$ and $N_{G^{k}}\left(C^{\prime}\right)=N_{G}(C) . G_{\alpha}^{k}\left(C^{\prime}\right)$ is connected (because for any vertices $x$ and $y$ in $C^{\prime}$, there is a path $P$ in $G(C)$ between $x$ and $y$ and by $(\mathrm{C})$, the sub-sequence of $P$ containing only the vertices belonging to $V_{\alpha}^{k}$ is a path in $G_{\alpha}^{k}\left(C^{\prime}\right)$ between $x$ and $y$ ). Let us show that $N_{G_{\alpha}^{k}}\left(C^{\prime}\right) \subseteq N_{G}(C)$. Let $x \in N_{G_{\alpha}^{k}}\left(C^{\prime}\right)$ and $y \in C^{\prime}$ such that $x y \in E_{\alpha}^{k}$. By (C), there is a path in $G$ between $x$ and $y$ all intermediate vertices of which belong to $V-V_{\alpha}^{k}$, and therefore belong to $V-S$ and consequently belong to $C$, so $x \in N_{G}(C)$. Let us show that $N_{G}(C) \subseteq N_{G^{k}}\left(C^{\prime}\right)$. Let $x \in N_{G}(C)$ and $y \in C$ such that $x y \in E$. As $C^{\prime} \neq \emptyset$, we may choose $z \in C^{\prime}$. Let $P$ be a path in $G(C)$ between $y$ and $z$ and let $z^{\prime}$ be the first vertex of $P$ from $y$ belonging to $V_{\alpha}^{k} . z^{\prime} \in C^{\prime}$ and by $(\mathrm{C}), x z^{\prime} \in E_{\alpha}^{k}$, so $x \in N_{G_{\alpha}^{k}}\left(C^{\prime}\right)$. Thus $N_{G_{\alpha}^{k}}\left(C^{\prime}\right)=N_{G}(C)$. As $C^{\prime} \neq \emptyset$, $C^{\prime} \subseteq V_{\alpha}^{k}-S, G_{\alpha}^{k}\left(C^{\prime}\right)$ is connected and $N_{G_{\alpha}^{k}}\left(C^{\prime}\right)=N_{G}(C) \subseteq S$, it follows that $C^{\prime} \in \mathcal{C}_{G_{\alpha}^{k}}(S)$. Therefore, $\left\{C \cap V_{\alpha}^{k}, C \in \mathcal{C}_{G}(S) \mid C \cap V_{\alpha}^{k} \neq \emptyset\right\} \subseteq \mathcal{C}_{G_{\alpha}^{k}}(S)$. As $\cup_{C \in \mathcal{C}_{G}(S)}\left(C \cap V_{\alpha}^{k}\right)=V_{\alpha}^{k}-S$, the reverse inclusion holds too.

Theorem 4.2: Let $\mathcal{S}$ be the set of substars of $(G, \alpha)$. Then any minimal triangulation $H$ of $G_{\S}$ is a minimal triangulation of $G$ which is a subgraph of $G_{\alpha}^{+}$.

Proof : We will use the following property $(\mathbf{P})$ proven in [4]:

(P) Let $\mathcal{S}$ be a set of pairwise non-crossing minimal separators of $G$ and $T$ be a minimal separator of $G_{\S}$. Then $\mathcal{C}_{G}(T)=\mathcal{C}_{G_{\S}}(T)$ and $\forall C \in \mathcal{C}_{G}(T), N_{G}(C)=N_{G_{8}}(C)$.

Let $H$ be a minimal triangulation of $G_{\delta}$. By Theorem 3.4 and Lemma 2.7, $H$ is a minimal triangulation of $G$. Let us show that $H$ is a subgraph of $G_{\alpha}^{+}$. By Theorem 2.4 and the fact that $G_{\delta}$ is a subgraph of $G_{\alpha}^{+}$, it is sufficient to show that any minimal separator of $G_{\delta}$ is a clique of $G_{\alpha}^{+}$. Let $T$ be a minimal separator of $G_{\delta}$ and $k$ be the minimum value of $\alpha(v)$ for $v$ in $T$. To show that $T$ is a clique of $G_{\alpha}^{+}$, it is sufficient to show that every vertex $v$ of $T-\left\{v_{k}\right\}$ is a neighbor of $v_{k}$ in $G_{\alpha}^{k}$ (because $N_{G_{\alpha}^{k}}\left(v_{k}\right)$ is a clique of $G_{\alpha}^{k+1}$ and therefore a clique of $G_{\alpha}^{+}$). Let $v \in T-\left\{v_{k}\right\}$. Let us show that $v \in N_{G_{\alpha}^{k}}\left(v_{k}\right)$. If there is a full component $C$ of $\mathcal{C}_{G}(T)$ such that $C \cap V_{\alpha}^{k}=\emptyset$ then let $P$ be a path in $G$ between $v_{k}$ and $v$ all intermediate vertices of which are in $C$; the intermediate vertices of $P$ do not belong to $V_{\alpha}^{k}$ so by $(\mathrm{C}) v \in N_{G_{\alpha}^{k}}\left(v_{k}\right)$. We suppose now that for every full component $C$ of $\mathcal{C}_{G}(T), C \cap V_{\alpha}^{k} \neq \emptyset$, and we assume by contradiction that $v \notin N_{G_{\alpha}^{k}}\left(v_{k}\right)$. As $T$ is a minimal separator of $G_{\delta}$, it is a minimal separator of $G$ by Theorem 3.4 and $(\mathrm{P})$ and therefore of $G_{\alpha}^{k}$ by Lemma 3.2. Let $S$ be the substar of $(G, \alpha)$ defined at step $k$ by the component containing $v$. $S$ is a minimal separator of $G_{\alpha}^{k}$ separating the vertices $v_{k}$ and $v$ of $T$, so $S$ and $T$ are crossing in $G_{\alpha}^{k}$. Hence $S$ intersects at least two components of $\mathcal{C}_{G^{k}}(T)$, and therefore of $\mathcal{C}_{G}(T)$ by Lemma 3.2 and of $\mathcal{C}_{G_{\delta}}(T)$ by Theorem 3.4 and (P). But as $S \in \mathcal{S}, S$ is a clique of $G_{\mathcal{S}}$ so that $S$ cannot intersect two components of $\mathcal{C}_{G_{s}}(T)$, a contradiction. 